\title{
Dynamic Mean-Field Glass Model with Reversible Mode Coupling and Trivial Hamiltonian
}

\author{
Kyozi Kawasaki + fi and Bongsoo Kim $\ddagger$ \\ $\dagger$ CNLS, Los Alamos National Laboratory, Los Alamos, NM 87545, USA \\ $\ddagger$ Physics Dept., Changwon National University, Changwon, 641-773, Korea
}

\begin{abstract}
Often the current mode coupling theory(MCT) of glass transitions is compared with mean field theories. We explore this possible correspondence. After showing a simple-minded derivation of MCT with some difficulties we give a concise account of our toy model developed to gain more insight into MCT. We then reduce this toy model by adiabatically eliminating rapidly varying velocitylike variables to obtain a Fokker-Planck equation for the slowly varying densitylike variables where diffusion matrix can be singular. This gives a room for nonergodic stationary solutions of the above equation.
\end{abstract}

\section{Introduction}

Our understanding of phase transition starts from the famous thesis of van der Waals of 1873 where complex effects of intermolecular interactions were put into two parameters often denoted as $b$ and $a$ representing, respectively, the repulsive and attractive parts of intermolecular forces. This theory was transcribed into magnetism resulting in the Weiss theory of ferromagnetism which contains a single parameter measuring strength of the molecular field. These mean field theories were remarkably successful and provided a good beginning of phase transition theory. Shortcomings of these theories were soon noticed, especially after Onsager's exact analysis of the two-dimensional Ising model. Thus the basis of the mean field theories were examined and exactly solvable model was constructed which yielded mean field theory results [1]. The characteristics of this model is that the attractive part of intermolecular potential has an infinite range after a certain limiting procedure, which suppress fluctuation effects responsible for deviations from the mean field behavior. Efforts to incorporate neglected fluctuation effects led to our current day understanding of phase transition, in particular, critical phenomena.

In comparison, the case of structual glass is much less clear-cut [2]. First there are still uncertainties about nature of the glass transition. It is not clear whether there is a real transition or just a cross-over. Eventhough one assumes a genuine transition, opinions differ among those who think that there is an underlying thermodynamic transition and those who believe that the transition is merely a kinetic one. The case of spin glass is much more well understood, at least on the mean field level where we know the existence of a genuine thermodynamic phase transition [3].

Under such circumstances it is quite significant that the first principle theory of structural glass transition, that is, the MCT, was proposed and succeeded in explaining

$\S$ Permanent address: 4-37-9 Takamidai, Higashi-ku, Fukuoka 811-0215, Japan 


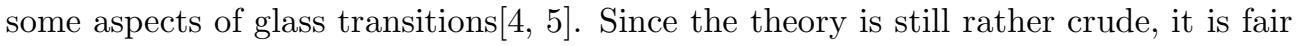
to regard the status of this theory as similar to that of van der Waals and Weiss theories mentioned above. As in the case of these theories the current MCT is beset with serious difficulties. These basically come from the fact that the MCT formalism was originally developed for critical phenomena focussing on very large length scales reaching to thousands of Ångstroms. Applications to glasses have difficulties due to its primarily short length scales and due to its own peculiarities. These difficulties are:

(a) The factorization approximation which replaces the four-body time corrrelation functions by the product of two-body time correlation functions is essential to obtain the self-consistent MCT equation. This is especially uncontrolled at short length scales of at most 10-20 Ångstroms. See the following section. (b) The idealized MCT predicts a sharp dynamic transition to a nonergodic state at a certain temperature. But MCT does not provide any information on the nature of this nonergodic state. (c) The physical picture of the so called hopping processes in an extended version of MCT is still lacking.

It should then be an urgent task for further progress to clarify the bases of the MCT. Motivated by this desire we constructed a toy model having the following three features [6]:

- reversible mode coupling mechanism

- trivial statics

- mean-field type so that the model can be exactly solvable.

This toy model is distinguished from other toy models for glasses in that it closely mimics the MCT 目目.

\section{MCT}

Here we present an over-simplified derivation of a self-consistent MCT equation of the density-density time correlation function obtained first in [4, 5]. We start from the following hydrodynamics-like continuum equation [7]:

$$
\begin{aligned}
& m \frac{\partial}{\partial t} \rho(\mathbf{r}, t)=-\boldsymbol{\nabla} \cdot \mathbf{j}(\mathbf{r}, t) \\
& \frac{\partial}{\partial t} \mathbf{j}(\mathbf{r}, t)=\mathbf{f}(\mathbf{r}, t)+\cdots
\end{aligned}
$$

Here $m$ is the mass of a fluid molecule, $\rho(\mathbf{r}, t)$ is the number density, and $\mathbf{j}(\mathbf{r}, t)$ is the momentum density. The ellipsis in (2) contains the terms second order in the momentum density, dissipative terms and thermal noise terms, which do not play a role here and will be dropped hereafter.

Here we assume the existence of a free energy density functional $H(\{\rho\})$. Then the body force density $\mathbf{f}(\mathbf{r}, t)$ is the number density times a force on a test particle of the same kind. The latter is negative of the gradient of the infinitesimal variation of the free energy density functional against infinitesimal density change, that is, $-\nabla \delta H(\{\rho\}) / \delta \rho(\mathbf{r})$. Therefore we find (Here time arguments are omitted.)

$$
\mathbf{f}(\mathbf{r})=-\rho(\mathbf{r}) \boldsymbol{\nabla} \delta H(\{\rho\}) / \delta \rho(\mathbf{r})
$$

Now, the exact form for $H(\{\rho\})$ is unknown and various approximate forms are proposed. For the purpose of deriving the MCT equation, it suffices to use the popular 
Ramakrishnan-Yussouf form despite its shortcomings [8]:

$$
\begin{aligned}
& H(\{\rho\})=k_{B} T \int d \mathbf{r} \rho(\mathbf{r})\left[\ln \left(\frac{\rho(\mathbf{r})}{\rho_{0}}\right)-1\right] \\
& -\frac{1}{2} k_{B} T \int d \mathbf{r} \int d \mathbf{r}^{\prime} c\left(\left|\mathbf{r}-\mathbf{r}^{\prime}\right|\right)\left(\rho(\mathbf{r})-\rho_{0}\right)\left(\rho\left(\mathbf{r}^{\prime}\right)-\rho_{0}\right)
\end{aligned}
$$

Here $\rho_{0}$ is the density of the reference uniform liquid and $c\left(\left|\mathbf{r}-\mathbf{r}^{\prime}\right|\right)$ is the direct correlation function [9] of the reference liquid. The Fourier transform $\hat{c}(\mathbf{k})$ of $c(r)$ is connected with the static structure factor of reference liquid $S(\mathbf{k})$ through

$$
\hat{c}(\mathbf{k})=\rho_{0}^{-1}-S(\mathbf{k})^{-1}
$$

The fact that the direct correlation function appears here is important because this is the only place in this theory where the short range correlation central to any liquid theory is incorporated.

The next step is to split $\mathbf{f}(\mathbf{r}, t)$ into terms linear and quadratic in the density difference $\delta \rho(\mathbf{r}, t) \equiv \rho(\mathbf{r}, t)-\rho_{0}$ as

$$
\begin{aligned}
& \mathbf{f}(\mathbf{r}, t)=\mathbf{f}^{l}(\mathbf{r}, t)+\mathbf{f}^{n l}(\mathbf{r}, t) \\
& \mathbf{f}^{l}(\mathbf{r}, t)=-\nabla p(\mathbf{r}, t) \\
& \mathbf{f}^{n l}(\mathbf{r}, t)=k_{B} T \int d \mathbf{r}^{\prime} c\left(\left|\mathbf{r}-\mathbf{r}^{\prime}\right|\right) \delta \rho(\mathbf{r}, t) \nabla^{\prime} \delta \rho\left(\mathbf{r}^{\prime}, t\right)
\end{aligned}
$$

where $p(\mathbf{r}, t)$ is the local pressure correct up to $\delta \rho(\mathbf{r}, \mathbf{t})$. Combination of (1),(2) and (6),(17) and (8) tells us that the $\mathbf{f}^{l}$ produces only linear density oscillations with constant wave vectors and does not contribute to freezing. On the other hand, $\mathbf{f}^{n l}(\mathbf{r}, t)$ is the sum of numerous terms oscillating with different frequencies, which, on the whole, look quite irregular. This fact also makes it hopeless to try to find solutions to these equations.

However, we are not interested in individual solutions but only some statistical properties of them, which are also measurable quantities. Among such quantities the most attention is paid to the density-density time correlation function, whose normalized form is defined in terms of $\rho_{\mathbf{k}}(t)$, the Fourier transform of $\delta \rho(\mathbf{r}, t)$, by

$$
\phi_{k}(t) \equiv \frac{<\rho_{\mathbf{k}}(t) \rho_{-\mathbf{k}}(0)>}{<\rho_{\mathbf{k}}(0) \rho_{-\mathbf{k}}(0)>}
$$

In obtaining this quantity from (11) and (2) we can regard $\mathbf{f}^{n l}(\mathbf{r}, t)$ as a kind of random force familiar in the Langevin equation of Brownian motion [10. Then the equation that determines $\phi_{k}(t)$ requires knowledge of a memory kernel which is the time correlation function of $\mathbf{f}_{\mathbf{k}}^{n l}(t)$, the Fourier transform of $\mathbf{f}^{n l}(\mathbf{r}, t)$.

Explicitly the equation for $\phi_{k}(t)$ turns out to be 11]

$$
\frac{d^{2} \phi_{k}(t)}{d t^{2}}=-\Omega_{k}^{2} \phi_{k}(t)-\int_{0}^{t} d s \mathcal{M}_{k}(t-s) \frac{d \phi_{k}(s)}{d s}
$$

where $\Omega_{k} \equiv k \sqrt{k_{B} T / S_{k}}$ is the frequency of the local density oscillation in liquids. $\mathcal{M}_{k}(t)$ is the memory kernel given by

$$
\mathcal{M}_{k}(t)=\frac{1}{m \rho_{0} k_{B} T k^{2} V}\left\langle f_{\mathbf{k}}^{n l}(t) f_{-\mathbf{k}}^{n l}(0)\right\rangle
$$

where $f_{\mathbf{k}}^{n l} \equiv i \mathbf{k} \cdot \mathbf{f}_{\mathbf{k}}{ }^{n l}$ and $V$ the system volume. Since this correlation function involves products of four density fluctuations which are impossible to deal with 
directly, this is factorized into products of two density-density corrrelation functions. This produces the feedback mechanism responsible for freezing. The resulting selfconsistent equation for the density-density correlation function is the same as that given for the first time in 1 . In the above simple derivation we have side-stepped the fact that the time dependence of $\mathbf{f}^{n l}(\mathbf{r}, t)$ in the memory kernel is in fact governed by the "projected" dynamics in the sense of Zwanzig-Mori formalism. The correct but more involved derivation starts from a Fokker-Planck type equation [11]. Alternative simpler derivation explicitly relies on the strong assumption that density fluctuations at various times obey Gaussian statistics [12].

The transition to non-ergodic states in this theory is driven by the nonlinear force term (8), which, in turn arises from the quadratic (or harmonic) term of (4). The first term there containing a logarithm is just for ideal gas. Therefore, the Hamiltonian of this theory does not require complex nonlinear terms that characterize many other theories or models of glass transitions.

\section{Mean field toy model}

\subsection{Model}

Our toy model is a set of oscillators with linear and random nonlinear couplings expressed by the following Langevin equations for the $N$-component density-like variables $a_{i}(t)$ with $i=1,2, \cdots, N$ and the $M$-component velocity-like variables $b_{\alpha}$ with $\alpha=i, 2, \cdots, M$. Here and after we will use Roman indices for the components of $a$ and Greek for those of $b$ :

$$
\begin{aligned}
& \dot{a}_{i}=K_{i \alpha} b_{\alpha}+\frac{\omega}{\sqrt{N}} J_{i j \alpha} a_{j} b_{\alpha} \\
& \dot{b}_{\alpha}=-\gamma b_{\alpha}-\omega^{2} K_{j \alpha} a_{j}-\frac{\omega}{\sqrt{N}} J_{i j \alpha}\left(\omega^{2} a_{i} a_{j}-T \delta_{i j}\right)+f_{\alpha} \\
& <f_{\alpha}(t)>=0, \quad<f_{\alpha}(t) f_{\beta}\left(t^{\prime}\right)>=2 \gamma T \delta_{\alpha \beta} \delta\left(t-t^{\prime}\right)
\end{aligned}
$$

where the summation is implied for repeated indices and overdots denote time derivatives. Here $\gamma$ is the decay rate of the velocity-like variables $b_{\alpha}$ and $\omega$ gives a measure of the frequencies of oscillations of the density-like variables $a_{j}$. The thermal noises $f_{\alpha}(t)$ are independent Gaussian random variables with zero mean and variance $2 \gamma T, T$ being the temperature of the heat bath with which the system has a thermal contact. The choice of this variance guarantees the proper equilibration of the variables $\{b\}$. The $N \times M$ matrix $K_{i \alpha}$ plays an important role in the model and for later purpose we impose the (one-sided) orthogonality

$$
K_{i \alpha} K_{i \beta}=\delta_{\alpha \beta}, \quad K_{i \alpha} K_{j \alpha} \neq \delta_{i j}
$$

where the last equation is due to the inequality $M<N$. For $M=N$ we can impose an additional condition $K_{i \alpha}=\delta_{i \alpha}$ and hence trivially $K_{i \alpha} K_{j \alpha}=\delta_{i j}$. We also note that $K_{i \alpha}$ governs linearized reversible dynamics of the model with the dynamical matrix $\Omega$ given by $\Omega_{i j} \equiv \omega^{2} K_{i \alpha} K_{j \alpha}$. The mode coupling coefficients $J_{i j \alpha}$ are chosen to be quenched (time-independent) Gaussian random variables with the following properties:

$$
\begin{aligned}
& {\overline{J_{i j \alpha}}}^{J}=0, \\
& {\overline{J_{i j \alpha} J_{k l \beta}}}^{J}=\frac{g^{2}}{N}\left[\left(\delta_{i k} \delta_{j l}+\delta_{i l} \delta_{j k}\right) \delta_{\alpha \beta}+K_{i \beta}\left(K_{k \alpha} \delta_{j l}+K_{l \alpha} \delta_{j k}\right)\right.
\end{aligned}
$$




$$
\left.+K_{j \beta}\left(K_{k \alpha} \delta_{i l}+K_{l \alpha} \delta_{i k}\right)\right]
$$

where $\bar{\cdots}^{J}$ denotes average over the $J$ 's. In constructing this model, we were motivated by the works 13, 14 in which random coupling models involving an infinite component order parameter have been shown to be exactly analyzed by mean-field-type concepts.

Equation (12) is analogous to the equation of continuity of fluid and (13) is like the equation of motion where the right hand side is like the force acting on a fluid element which corresponds to (11) and (2), respectively. We will eventually take $N$ and $M$ infinite with the ratio $\delta^{*} \equiv M / N$ kept finite.

One can derive from the Langevin equations (1)-(3) the corresponding FokkerPlanck equation for the probability distribution function $D(\{a\},\{b\}, t)$ for our variable set denoted as $\{a\},\{b\}$ as follows

$$
\partial_{t} D(\{a\},\{b\}, t)=\hat{L} D(\{a\},\{b\}, t)
$$

where the Fokker-Planck operator is given by $\hat{L}=\hat{L}_{0}+\hat{L}_{1}+\hat{L}_{M C}$ with

$$
\begin{aligned}
& \hat{L}_{0} \equiv \frac{\partial}{\partial b_{\alpha}} \gamma\left(T \frac{\partial}{\partial b_{\alpha}}+b_{\alpha}\right), \quad \hat{L}_{1} \equiv K_{j \alpha}\left(-\frac{\partial}{\partial a_{j}} b_{\alpha}+\frac{\partial}{\partial b_{\alpha}} \omega^{2} a_{j}\right), \\
& \hat{L}_{M C} \equiv \frac{1}{\sqrt{N}} J_{i j \alpha}\left(-\frac{\partial}{\partial a_{i}} \omega a_{j} b_{\alpha}+\frac{\partial}{\partial b_{\alpha}} \omega\left(\omega^{2} a_{i} a_{j}-T \delta_{i j}\right)\right)
\end{aligned}
$$

It is then easy to show that the equilibrium stationary distribution (i.e., $\hat{L} D_{e}(a, b)=0$ ) is given by

$$
D_{e}(\{a\},\{b\})=\text { cst. } \mathrm{e}^{-\sum_{j=1}^{N} \frac{\omega^{2}}{2 T} a_{j}^{2}-\sum_{\alpha=1}^{M} \frac{1}{2 T} b_{\alpha}^{2}}
$$

where $c$ st. is the normalization factor.

\subsection{Analysis and discussion}

We aim at finding the set of five equilibrium time correlation functions defined by

$$
\begin{gathered}
C_{a}\left(t-t^{\prime}\right) \equiv \frac{1}{N}<a_{j}(t) a_{j}\left(t^{\prime}\right)>, \quad C_{a b}\left(t-t^{\prime}\right) \equiv \frac{1}{M} K_{j \alpha}<a_{j}(t) b_{\alpha}\left(t^{\prime}\right)>, \\
C_{b a}\left(t-t^{\prime}\right) \equiv \frac{1}{M} K_{j \alpha}<b_{\alpha}(t) a_{j}\left(t^{\prime}\right)>, \quad C_{b}\left(t-t^{\prime}\right) \equiv \frac{1}{M}<b_{\alpha}(t) b_{\alpha}\left(t^{\prime}\right)>, \\
C_{a}^{K}\left(t-t^{\prime}\right) \equiv \frac{1}{M} K_{i \alpha} K_{j \alpha}<a_{i}(t) a_{j}\left(t^{\prime}\right)>
\end{gathered}
$$

It turns out that we need to have the last correlation function to close the selfconsistent set of equations for the correlators when $M<N$. Note that for the case $M=N$, we can take $K_{i \alpha}=\delta_{i \alpha}$, and then $C_{a}^{K}\left(t-t^{\prime}\right)=C_{a}\left(t-t^{\prime}\right)$.

In order to obtain this self-consistent set of equations, it is most convenient to adapt the generating functional method from which one can write down the set of effective linear Langevin equations valid in the limit of $M, N \rightarrow \infty$. We refer 17 for further details. ¿From this effective Langevin equations, one can readily derive the following closed self-consistent equations for $t>0$ for the five correlators:

$$
\begin{aligned}
& \dot{C}_{a}(t)=\delta^{*} C_{b a}(t)-\Sigma_{a a} \otimes C_{a}(t)-\delta^{*} \Sigma_{a b} \otimes C_{b a}(t) \\
& \dot{C}_{b a}(t)=-\gamma C_{b a}(t)-\omega^{2} C_{a}^{K}(t)-\Sigma_{b a} \otimes C_{a}^{K}(t)-\Sigma_{b b} \otimes C_{b a}(t) \\
& \dot{C}_{a b}(t)=C_{b}(t)-\Sigma_{a a} \otimes C_{a b}(t)-\Sigma_{a b} \otimes C_{b}(t), \\
& \dot{C}_{b}(t)=-\gamma C_{b}(t)-\omega^{2} C_{a b}(t)-\Sigma_{b a} \otimes C_{a b}(t)-\Sigma_{b b} \otimes C_{b}(t) \\
& \dot{C}_{a}^{K}(t)=C_{b a}(t)-\Sigma_{a a} \otimes C_{a}^{K}(t)-\Sigma_{a b} \otimes C_{b a}(t)
\end{aligned}
$$


where, for any function $X(t), X \otimes a(t) \equiv \int_{-\infty}^{t} d t^{\prime} X\left(t-t^{\prime}\right) a\left(t^{\prime}\right)$. The equations (21)(25) constitute the self-consistent equations for the 5 correlators $C_{a}(t), C_{b a}(t), C_{a b}(t)$, $C_{b}(t)$, and $C_{a}^{K}(t)$. This set of equations can be solved numerically with the initial conditions $C_{a}(0)=C_{a}^{K}(0)=T / \omega^{2}, C_{a b}(0)=C_{b a}(0)=0$, and $C_{b}(0)=T$. Here the kernels $\Sigma$ 's are given by

$$
\begin{aligned}
\Sigma_{a a}\left(t-t^{\prime}\right) \equiv \delta^{*} & \frac{g^{2} \omega^{4}}{T}\left(C_{a}\left(t-t^{\prime}\right) C_{b}\left(t-t^{\prime}\right)+\delta^{*} C_{a b}\left(t-t^{\prime}\right) C_{b a}\left(t-t^{\prime}\right)\right), \\
\Sigma_{a b}\left(t-t^{\prime}\right) & \equiv-2 \delta^{*} \frac{g^{2} \omega^{4}}{T} C_{a}\left(t-t^{\prime}\right) C_{b a}\left(t-t^{\prime}\right) \\
\Sigma_{b a}\left(t-t^{\prime}\right) & \equiv-2 \delta^{*} \frac{g^{2} \omega^{6}}{T} C_{a}\left(t-t^{\prime}\right) C_{a b}\left(t-t^{\prime}\right), \\
\Sigma_{b b}\left(t-t^{\prime}\right) & \equiv \frac{2 g^{2} \omega^{6}}{T} C_{a}\left(t-t^{\prime}\right)^{2}
\end{aligned}
$$

These kernels arise from the non-linear mode coupling terms in (17) and (18). Note that the correlator $C_{a}^{K}\left(t, t^{\prime}\right)$ is not involved in the $\Sigma$ 's.

For further analyses it is very convenient to work with the equations of the Laplace transformed correlation functions defined as $C^{L}(z) \equiv \int_{0}^{\infty} d t \mathrm{e}^{-z t} C(t)$. Performing the Laplace transformation of the self-consistent equations and rearranging them we obtain

$$
\begin{aligned}
C_{a}^{L}(z)=\frac{T}{\omega^{2}} \frac{1}{z+\Sigma_{a a}^{L}(z)}[ & \left.1-\delta^{*} \frac{\omega^{2}\left(1-\Sigma_{a b}^{L}(z)\right)^{2}}{\left(z+\Sigma_{a a}^{L}(z)\right)\left(z+\gamma+\Sigma_{b b}(z)\right)+\omega^{2}\left(1-\Sigma_{a b}^{L}(z)\right)^{2}}\right] \\
C_{a b}^{L}(z) & =-\frac{T\left(1-\Sigma_{a b}^{L}(z)\right)}{\left(z+\Sigma_{a a}^{L}(z)\right)\left(z+\gamma+\Sigma_{b b}(z)\right)+\omega^{2}\left(1-\Sigma_{a b}^{L}(z)\right)^{2}} \\
C_{b a}^{L}(z) & =\frac{T\left(1-\Sigma_{a b}^{L}(z)\right)}{\left(z+\Sigma_{a a}^{L}(z)\right)\left(z+\gamma+\Sigma_{b b}(z)\right)+\omega^{2}\left(1-\Sigma_{a b}^{L}(z)\right)^{2}} \\
C_{b}^{L}(z) & =\frac{T\left(z+\Sigma_{a a}^{L}(z)\right)}{\left(z+\Sigma_{a a}^{L}(z)\right)\left(z+\gamma+\Sigma_{b b}(z)\right)+\omega^{2}\left(1-\Sigma_{a b}^{L}(z)\right)^{2}} \\
C_{a}^{K L}(z) & =\frac{T}{\omega^{2}}\left[z+\Sigma_{a a}^{L}(z)+\frac{\omega^{2}\left(1-\Sigma_{a b}^{L}(z)\right)^{2}}{z+\gamma+\Sigma_{b b}(z)}\right]^{-1}
\end{aligned}
$$

For $\delta^{*}=1$ where $M=N$ and $K_{i \alpha}=\delta_{i \alpha}, C_{a}^{L}(z)=C_{a}^{K L}(z)$ reproduces the equation derived in [15], apart from the wave number dependence. Note that if we put $\Sigma_{a a}^{L}(z)=\Sigma_{a b}^{L}(z)=0$ by hand, (27) or (31) gives a closed equation for $C_{a}(t)$ alone. This equation is nothing but the Leutheusser's schematic MC equation giving a dynamic transition from ergodic phase to nonergodic one 佃. But in reality $\Sigma_{a a}$ and $\Sigma_{a b}$ can not be ignored a priori and our numerical solution strongly indicates that the system remains ergodic for all temperatures due to the strong contribution of these so called hopping terms. Furthermore these hopping terms cannot be made selfconsistently small as temperature is lowered. Therefore the density correlator does not show a continuous slowing down with lowering temperature. This result was striking to us since usually a mean-field-type theory, such as the dynamics of the spherical $p$-spin model in the limit of $N \rightarrow \infty$, often gives a sharp dynamic transition [16].

Thus it is very difficult to understand the idealized MCT without relying upon uncontrolled approximations. It is also interesting to note that the ergodicity restoring process in our toy model (represented by the kernels $\Sigma_{a a}$ and $\Sigma_{a b}$ ) has nothing to do with a thermally activated energy barrier crossing since the quadratic Hamiltonian in our model does not possess such a barrier. 


\section{Reduced Fokker-Planck equation for the density-like variables}

Possibility of nonergodic states in our model can be seen more directly by adiabatically eliminating the variables $\{b\}$ in the limit of large $\gamma$ and obtaining the reduced Fokker-Planck equation for the distribution function $\tilde{D}(\{a\}, t)$ containing only the $\{a\}$ variables:

$$
\begin{aligned}
& \frac{\partial \tilde{D}(\{a\}, t)}{\partial t}=\mathcal{L}_{F P} \tilde{D}(\{a\}, t) \\
& \equiv \frac{\partial}{\partial a_{i}} Q_{i j}(\{a\})\left(\frac{\partial}{\partial a_{j}}+\frac{\omega^{2}}{T} a_{j}\right) \tilde{D}(\{a\}, t)
\end{aligned}
$$

where we have defined the Fokker-Planck operator $\mathcal{L}_{F P}$ through the second member of the above equation. Here the diffusion matrix $Q_{i j}(\{a\})$ is given by

$$
\begin{aligned}
& Q_{i j}(\{a\}) \equiv \frac{T}{\gamma} M_{i \alpha} M_{j \alpha} \\
& M_{i \alpha} \equiv K_{i \alpha}+\frac{\omega}{\sqrt{N}} J_{i k \alpha} a_{k}
\end{aligned}
$$

The positive semi-definiteness of the diffusion tensor $\mathbf{Q}$ is very easy to show because for an arbitrary $N$ component real vector we have

$$
x_{i} Q_{i j} x_{j}=\frac{T}{\gamma} \sum_{\alpha}\left(M_{i \alpha} x_{i}\right)^{2} \geq 0
$$

The crucial point is that the diffusion matrix $Q_{i j}$ is singular for $M<N$, i.e., $\operatorname{det}|\mathbf{Q}|=0$ giving rise to zero eigenvalues for $\mathbf{Q}$ [17]. This implies that the FokkerPlanck equation (32) can have nonequilibrium stationary solution other than the equilibrium one, $D_{e}(\{a\})=c s t \cdot \exp \left(-\omega^{2} a_{j}^{2} / 2 T\right)$. This nonequilibrium stationary solutions are precisely the kind of nonergodic states found numerically in the present toy model. In fact a class of stationary solutions is given by

$$
\tilde{D}_{s}(\{a\})=\mathcal{F}\left(\xi_{j} a_{j}\right) \mathrm{e}^{-\frac{\omega^{2}}{2 T} a_{i}^{2}}
$$

where $\xi_{i}$ is an eigenvector of the diffusion matrix $Q_{i j}$ with zero eigenvalue and $\mathcal{F}(x)$ a non-negative function. If the function $\mathcal{F}(x)$ is a constant, then $\tilde{D}_{s}(\{a\})=\tilde{D}_{e}(\{a\})$ is the equilibrium distribution, otherwise it is a nonequilibrium stationary distribution. Hence the model is nonergodic for $0 \leq \delta^{*}<1$.

Let us now investigate this point somewhat further. We first define the following non-negative ratio:

$$
R(\{a\}, t) \equiv \frac{\tilde{D}(\{a\}, t)}{\tilde{D}_{e}(\{a\})}
$$

and then introduce a Boltzmann's H-like quantity as

$$
\mathcal{H}(t) \equiv \int d\{a\} \tilde{D}(\{a\}, t) \ln R(\{a\}, t)
$$

where the integration is over all the variables in the set $\{a\}$. Using normalization property of the distribution function and integrating by parts assuming a natural boundary condition we find

$$
\dot{\mathcal{H}}(t)=\int d\{a\} \tilde{D}(\{a\}, t) \mathcal{L}_{F P}^{\dagger}(\{a\}) \ln R(\{a\}, t)
$$


with $\mathcal{L}_{F P}^{\dagger}(\{a\})$ the adjoint operator of $\mathcal{L}_{F P}(\{a\})$ :

$$
\mathcal{L}_{F P}^{\dagger}(\{a\}) \equiv\left(\frac{\partial}{\partial a_{i}}-\frac{\omega^{2}}{T} a_{i}\right) Q_{i j}(\{a\}) \frac{\partial}{\partial a_{j}}
$$

Now we readily verify that

$$
\mathcal{L}_{F P}^{\dagger}(\{a\}) \ln R(\{a\}, t)=\frac{1}{R} \mathcal{L}_{F P}^{\dagger} R-Q_{i j} \frac{1}{R} \frac{\partial R}{\partial a_{j}} \frac{1}{R} \frac{\partial R}{\partial a_{i}}
$$

Substituting this into (39) we finally find

$$
\dot{\mathcal{H}}(t)=-\int d\{a\} \tilde{D}(\{a\}, t) Q_{i j}(\{a\}) \frac{\partial \ln R(\{a\}, t)}{\partial a_{i}} \frac{\partial \ln R(\{a\}, t)}{\partial a_{j}}
$$

where the contribution of the first term in (41) vanishes since

$$
\int d\{a\} \frac{\tilde{D}}{R} \mathcal{L}_{F P}^{\dagger} \cdots=\int d\{a\} \tilde{D}_{e} \mathcal{L}_{F P}^{\dagger} \cdots=\int d\{a\}\left(\mathcal{L}_{F P} \tilde{D}_{e}\right) \cdots=0
$$

This result shows a kind of Boltzmann's H-theorem [18] (See [10] for a discussion for general master equations with detailed balance.):

$$
\dot{\mathcal{H}}(t) \leq 0
$$

Let us suppose that we have performed a transformation of the variables $\{a\} \rightarrow$ $\{s\}, \tilde{D}(\{a\}) d\{a\} \rightarrow \hat{D}(\{s\}) d\{s\}, R(\{a\}) \rightarrow \hat{R}(\{s\})$ etc. so that the matrix $Q_{i j}(\{a\})$ is diagonalized:

$$
\hat{Q}_{i j}(\{s\})=\lambda_{i}(\{s\}) \delta_{i j}
$$

with the eignevalues $\lambda_{i}(\{s\})$ non-negative functions of $\{s\}$. For the case of diagonalized $Q_{i j}$, (44), the results (42) and (43) become

$$
\int d\{s\} \sum_{i} \lambda_{i}(\{s\}) \hat{D}(\{s\}, t)\left(\frac{\partial \ln \hat{R}(\{s\}, t)}{\partial s_{i}}\right)^{2} \geq 0
$$

The stationarity condition $\frac{\partial}{\partial t} \hat{D}(\{s\}, t)=0$ or $\dot{\mathcal{H}}(t)=0$ then implies

$$
\int d\{s\} \sum_{i} \lambda_{i}(\{s\}) \hat{D}(\{s\}, t)\left(\frac{\partial \ln \hat{R}(\{s\}, t)}{\partial s_{i}}\right)^{2}=0
$$

If we denote those subset of the variables of $\{s\}$ with positive eigenvalues as $s_{\alpha}, \alpha=$ $1,2, \cdots, M^{\prime}(\leq M)$, which we denote as $\{s\}^{\prime}$, we must have in the region $\hat{D}(\{s\}, t)>0$,

$$
\frac{\partial \ln \hat{R}(\{s\}, t)}{\partial s_{\alpha}}=0 \text { for } \lambda_{\alpha}(\{s\})>0
$$

We note that validity of the condition (47) in general depends on the regions in the space of the variables $\{s\}$ through $\{s\}$ dependence of the $\lambda$ 's. Thus, in a particular region in which $\lambda_{\alpha}(\{s\}) \hat{D}(\{s\})$ are positive definite, $R(\{s\}, t)$ does not contain $s_{\alpha}$. If we denote the remaining set of the variables $\{s\}$ than those corresponding to positive eigenvalues as $u_{\beta}, \beta=M^{\prime}+1, M^{\prime}+2, \cdots, N$, which are corresponding to the zero eigenvalues, we should have $\hat{R}(\{s\}, t)=\hat{R}\left(\{s\}^{\prime},\{u\}, t\right)=\hat{R}(\{u\}, t)$. Consequently, from (37), the general form of non-equilibrium stationary state distribution function is

$$
\hat{D}_{S}\left(\{s\}^{\prime},\{u\}\right)=\hat{R}_{S}(\{u\}) \hat{D}_{e}\left(\{s\}^{\prime},\{u\}\right)
$$

The previous result, (36), is a special case of this general form. 
Glass Model with Mode Coupling and Trivial Hamiltonian

\section{Summary and discussion}

Our toy model analyses show that the so-called hopping processes introduced as a correction to the idealized MCT [5] are the effects of the velocity-like variables, which still enter in the framework of the meanfield treatment, and are not directly related to the barrier-crossing mechanism. In our toy model, strength of the hopping processes can be adjusted through the parameter $\delta^{*}$. Possible nonequilibrium stationary states are connected to the singularity of the diffusion matrix of reduced Fokker-Planck equation of Section 1 involving only the varaibles $\{a\}$. This feature, suitably extended to general master equation, would be common to many kinetically constrained glass transition models [19] and is worth further exploration.

\section{Acknowledgments}

We thank K. Dawson, M. Fisher, W. Götze, J. Jäckle, A. Latz, S. J. Lee, M. Mézard, R. Schilling and C. Tsallis for useful suggestions and discussions. The research of KK is supported by the Department of Energy, under contract W-7405-ENG-36. An additional partial support to KK by the Cooperative Research under the Japan-U.S. Cooperative Science Program sponsored by Japan Society of Promotion of Science is also gratefully aknowledged. BK is supported by the Interdisciplinary Research Program of the KOSEF (Grant No. 1999-2-114-007-3).

\section{References}

[1] Kac M, Uhlenbeck G E, and Hemmer P C J. Math. Phys. 196193216

[2] Jäckle J 1986 Rep. Prog. Phys. 49171

[3] Mézard M, Parisi G, and Virasoro M A 1987 Spin Glass Theory and Beyond (World Scientific: Singapore)

[4] Bentzelius U, Götze W, and Sjölander A 1984 J. Phys. C 175915 Leutheusser E 1984 Phys. Rev. A 292765

[5] Götze W 1991 Liquids, Freezing and Glass Transition, eds Hansen J, Levesque D, and ZinnJustin J (North-Holland: Amsterdam); Götze W and Sjögren L 1992 Rep. Prog. Phys. 55 241; 1995 Transport Theor. Stat. Phys. 24801

Kim B and Mazenko G F 1990 Advances in Chemical Physics 78129.

More recent developments can be found in the collection of papers in Yip Y (guest ed) 1995 Relaxation Kinetics in Supercooled Liquids-Mode Coupling Theory and its Experimental Tests; Transport Theor. Stat. Phys. 24 Nos 6-8 and the conference proceedings such as 1998 J. Non-cryst. Solids 235-237 and 1999 J. Phys.: Condens. Matter 10A

[6] Kawasaki K and Kim B 2001 Phys. Rev. Lett. 863582

[7] Das S and Mazenko G F 1986 Phys. Rev. A 342265

[8] Ramakrishnan T V and Yussouff M 1979 Phys. Rev. B 192775

[9] Hansen J P, and McDonald I R 1986 Theory of Simple Liquids 2nd ed (Academic Press: London)

[10] van Kampen N G 1992 Stochastic Processes in Physics and Chemistry (North-Holland: Amsterdam)

[11] Kawasaki K 2000 Recent Res. Devel. Stat. Phys. 141

[12] Zaccarelli E, Foffi G, Sciortino F, Tartaglia P and Dawson K A 2001 Europhys. Lett. 55, 157

[13] Kraichnan R H 1959 J. Fluid Mech. 532

[14] Bouchaud J -P, Cugliandolo L, Kurchan J, and Mézard M 1996 Physica 226A 243; 1998 Spin Glasses and Random Fields ed A P Young (World Scientific: Singapore )

[15] Schmitz R, Dufty J W and De P 1993 Phys. Rev. Lett. 712066

[16] Crisanti A, Horner H, and Sommers H J 1993 Z. Phys. B 92257

[17] Kim B and Kawasaki K, J. Phys. Condens. Matter (submitted)

[18] Risken H 1989 The Fokker-Planck Equation (Springer Verlag: Heidelberg)

[19] Proceedings of the Barcelona worshop on Glassy behavior of kinetically constrained models edited by Ritort F and Sollich P, 2002 J. Phys.: Condens. Matter 